\title{
Comparison of the Spinal Loads Produced by Carrying a Backpack and Carrying a Person Piggyback: Crossfit Training
}

\author{
Sheena Graham ${ }^{1}$, Marion J.L. Alexander ${ }^{1}$, Jeff Leiter ${ }^{2}$, Cheryl Glazebrook ${ }^{1}$, David Telles-Langdon ${ }^{3}$ \\ ${ }^{I}$ Faculty of Kinesiology and Recreation Management, University of Manitoba, Winnipeg, Manitoba, Canada R3T 2N2 \\ ${ }^{2}$ Pan Am Clinic75 Poseidon Bay, Winnipeg, Manitoba, R3M 3E4, Canada \\ ${ }^{3}$ Gupka Faculty of Kinesiology and Sport Science, University of Winnipeg \\ Corresponding Author: Marion J.L. Alexander, E-mail: Marion.Alexander@umanitoba.ca
}

\section{ARTICLE INFO}

Article history

Received: August 09, 2018

Accepted: October 26, 2018

Published: October 31, 2018

Volume: 6 Issue: 4

Conflicts of interest: None

Funding: None

\begin{abstract}
Background of Study: The piggyback carry has recently become a more popular exercise through the emerging sport of CrossFit. Purpose: The purpose of this study was to determine any biomechanical differences that exist in the lumbar spine when carrying no load, a backpack, and a person on the back. Methods: Twelve 70+ kg male strength-trained athletes were recruited from local CrossFit affiliates. One child with a mass of $27 \mathrm{~kg}$ was recruited to be the piggyback passenger for all participants. All participants and the guardian of the passenger signed an informed consent form. The participants walked three times over a force plate for each of three conditions: carrying no load, a $27 \mathrm{~kg}$ backpack, or a $27 \mathrm{~kg}$ passenger. Three Canon video cameras recorded each trial, and Dartfish Software was used to measure joint angles and gait parameters. Maximal trunk inclination angle, was used in a static lumbar spine model to calculate trunk muscle torque and force, and lumbosacral joint reaction forces. Results: Both load conditions produced compensatory trunk flexion; trunk flexion increased from no load to piggybacking to backpacking. Mean values were determined for each participant for each variable, and these values were compared amongst the three conditions of no load, piggybacking, and backpacking. An alpha value of 0.05 was used. Conclusion: Due to the more extreme position of the trunk and greater magnitude of torques, backpacking likely places the musculoskeletal system at more risk than does piggybacking.
\end{abstract}

Key words: Lumbar Vertebrae/Physiology, Torque, Weight-bearing, Lifting/Adverse Effects

\section{INTRODUCTION}

Carrying a load, and specifically carrying a load posterior to the spine, has been identified as an activity that should be considered as a possible risk to the lumbar spine by lifting studies (Cholewicki, McGill, \& Norman, 1991) and backpack studies (Chow et al., 2005; Goh, Thambyah, \& Bose, 1998). Addressing the trunk flexion angle and estimating the compressive, shear, and resultant joint reaction forces at the lumbosacral joint during this activity will help to describe such risk. Most North Americans have likely either been carried by another person, have carried another person, or have experienced both roles in the execution of a piggyback (Figure 1). The piggyback is not only an important part of family life and social culture, as a common bonding and rough-housing experience important to social and physical development within a family or friendship (Bennett, 1999), but it is also used by athletes for strength training. This skill has been used for resistance training by boot camps, soccer teams and rugby teams (Piggyback New West, 2013; Rugby, 2011; Sportsmail Reporter, 2011) and has become a larger part of the athletic community through the recent trend of CrossFit training (Crossfit Inc, 2008).
During CrossFit training, athletes perform a variety of exercises that could be described as a combination of activities of daily living and resistance training, generally with the goal of surpassing previous self-perceived limits of physical capability. Examples of exercises are walking lunges, pull-ups, tire lifts, the kettle bell snatch (Crossfit Winnipeg, 2014) and variations of the piggyback (Crossfit Inc, 2008). The CrossFit piggyback may be performed while standing, walking, or during other activities such as calf raises or squats to add load for more resistance. The piggyback was also included in a timed circuit as the 50 yard piggyback dash for example. The creator of that specific workout of the day (WOD) allowed a loaded backpack to be substituted for the person during the piggyback portion of the challenge

\section{Description of the Skill}

During the piggyback, the body of the passenger is posterior to and wrapped around the carrier (Figure 1); the arms wrap over the shoulders and anteriorly around the neck while the legs wrap laterally and are held by the carrier superior to the 
iliac crest. The weight of the passenger is supported partly by the carrier exerting a lifting force on the legs of the passenger and the friction and weight of the passenger's pelvis resting on the lower back of the carrier, and the arms of the passenger exerting a downward force on the shoulder girdle of the carrier. The passenger can help support more of his or her weight by exerting a greater force on the shoulder girdle of the carrier, but the entire weight of the passenger is basically supported by the carrier superior to the lumbosacral joint. For the purposes of this study, the trunk will be considered to be in an approximately constant position of flexion during the piggyback while the legs transport the body across the ground.

\section{Piggybacking}

The piggyback carry has been referenced in several popular culture articles, some of which acknowledged there are safety issues associated with the piggyback carry. A mother wrote to a Doctor's column asking if it was safe for her daughter's high school soccer team to train by carrying each other on the back (Donohue, 1996). The doctor wrote that this exercise was not advised as it involved a lot of shifting dead weight carried on the back, and that it could possibly disrupt natural stride and balance to increase the risk of injury to the back and legs. A fitness article in the Washington

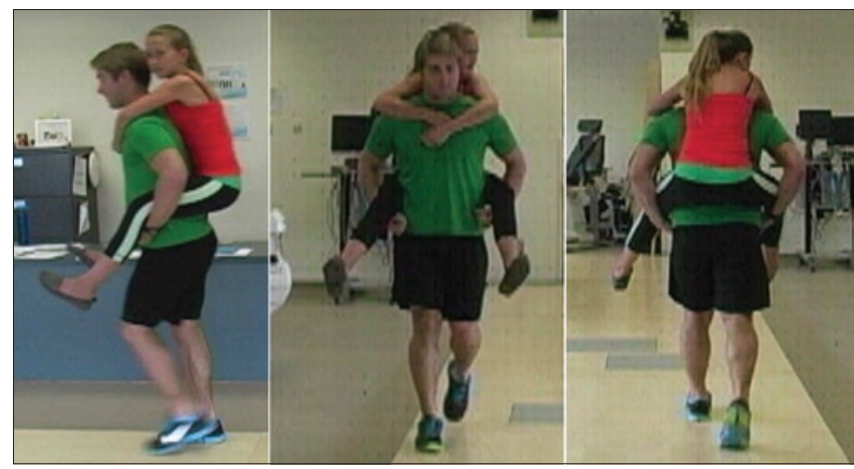

Figure 1. The piggyback carry from sagittal, anterior and posterior views
Post suggested performing squats while piggybacking as part of a romantic partner exercise routine (Schneider, 2009). Between squats, the carrier was instructed to walk ten to twenty feet. When participating in this exercise, one partner would likely be significantly heavier than the other. Without previous training, this may be a risky activity for the person that weighed less, but the author does not describe any possibility of risk (Schneider, 2009). This study will address the trunk position during piggyback and backpack carriage and then estimate the lumbosacral forces present during these types of posterior load. If the trunk did not adjust into a flexed position to move the centre of gravity of the backpack or piggyback load forward to be within the base of support of the carrier, the load would create an extensor torque. Therefore, the piggyback is an interesting exercise that necessitates the coordination of the trunk flexors, which resist the extensor torque initially caused by the load to flex the trunk and pull the line of gravity anteriorly to stay within the base of support, and the trunk extensors, which prevent the trunk from collapsing into further flexion due to the force of gravity on the upper body once it has been moved anteriorly.

\section{Trunk Flexion}

This important concept, previously described by Goh (Goh et al., 1998) and illustrates compensatory trunk flexion posture when carrying a load posterior to the trunk (Figure 2). This diagram illustrates how adding a loaded backpack moves the centre of gravity of the body posteriorly. In the following portion of the diagram, the trunk moves into a position of flexion to shift the centre of gravity of the body anteriorly back to its original position over the base of support at the feet (Goh et al., 1998). Based on this idea, it is thought that the current backpack condition will result in a position of greater trunk flexion than the current piggyback condition. Unloaded, the centre of gravity is near the anterior-posterior centre of the body within the inferior torso (Goh et al., 1998). The magnitudes of the calculated forces and torques at L5/S1 and trunk flexion angles may be related to lumbar

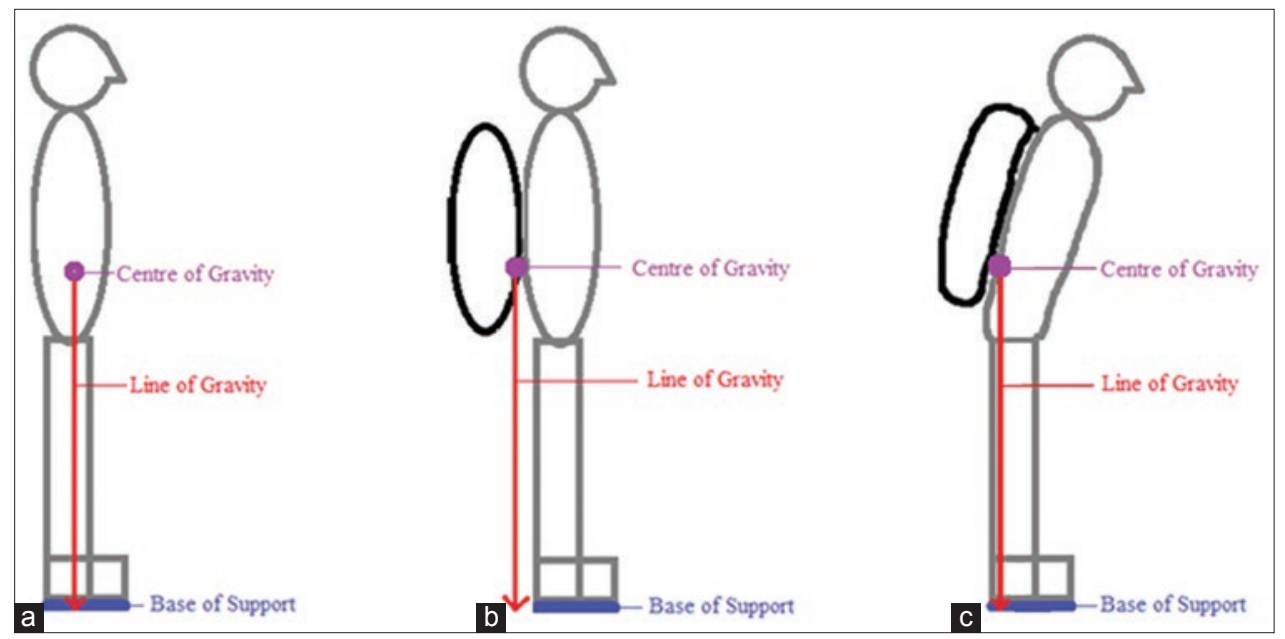

Figure 2. Trunk flexion is a response to the addition of a posterior load. a) The centre of gravity of the system is naturally located above the base of support. b) The addition of a posterior load shifts the centre of gravity of the system posteriorly, bringing the line of gravity potentially out of the base of support. c) The trunk flexes in order to shift the centre of gravity of the system forward and over the base of support. (Goh et al., 1998) 
spine risk between conditions. Although lumbar spine risk should be considered in examining piggyback loads, it must be remembered that this exercise is being performed in order to strengthen the spine and the core muscles in order to improve fitness and performance. The ground reaction forces were also estimated and compared in each of the three situations. This process enabled the researchers to determine the relative safety of the piggyback carry compared to the backpack carry, both of which are used in physical training as used in the sport of Crossfit.

\section{METHODS}

The main purpose of this study was to compare the forces and torques applied to the lumbar spine during three conditions: normal walking, carrying a backpack and carrying a passenger by piggyback. A biomechanical model was devised which accepted as input the torques applied in the clockwise and counterclockwise directions about the L5/S1 axis, and used these values to determine the total torques acting in each condition, as well as the total compressive and shear forces acting on $\mathrm{S} 1$.

\section{Participants and Design of Study}

The key problem examined in this study was to determine the whether the forces and torques on the lumbar spine differ when carrying no load, a backpack or a passenger of the same weight. A cross-sectional, descriptive design was used to quantify lumbar forces and torques produced in three different loading conditions in a laboratory setting. The order of the BP and PB loads were randomized while using force plate analysis and two dimensional motion capture analysis to quantify the forces and torques acting at the L5/S1 junction.

An ANOVA was used to determine the differences between the spinal loads experienced by the subjects in each of these load conditions. The participants included twelve fit adult males with an average mass of $82.4 \pm 8.3 \mathrm{~kg}$ volunteered as load carrier participants. Calculated for the use of an ANOVA with an alpha value of 0.05 , an effect size of 0.4 , three groups, three measurements, and a 0.5 correlation among repeated measures, G*power software determined that twelve participants were necessary for a power level of 0.80 (Faul, Erdfelder, Lang, \& Buchner, 2007). The results of a similar study, which compared trunk flexion between walking with a posterior $(15 \mathrm{~kg})$ backpack load and walking with a mostly posterior $(12 \mathrm{~kg})$ but partly anterior (3 kg) backpack load, were considered when making this calculation (Gillet, Foissac, Leteneur, Freychat, \& Babier, 2006). These authors measured trunk flexion angles of $14.2 \pm 3.2^{\circ}$ for the posterior backpack load and $10.6 \pm 2.9^{\circ}$ for the partly anterior load. This study compared the forces and torques on the lumbar spine in fit males while unloaded, carrying a passenger, and carrying a $29 \mathrm{~kg}$ backpack.

All participants were resistance-trained fit adult males, and nine of the twelve were CrossFit athletes. Recruitment was aimed at CrossFit males who had used the piggyback carry, but the criteria for inclusion were: a male athlete with a body mass of at least $70 \mathrm{~kg}$, regular physical training that involved lifting or carrying a minimum of $40 \mathrm{~kg}$ at least once per week, and no musculoskeletal injury within the last six months. A 29 kilogram female child, who was located through family friends, was used as the passenger load for the piggyback condition.

\section{Weight of the Load}

One goal of the present study was to use the heaviest load that was not considered a high risk to the carriers. Based on the more difficult load-carrying tasks and much heavier loads carried in related studies ((Beekley, 2007; Birrell, Hooper, \& Haslam, 2007; Grenier et al., 2012)), it was considered reasonable to ask these weight-trained participants to walk less than ten meters several times while carrying twenty nine kilograms of load. It was determined that a heavier subject might pose an injury risk to the participants and a heavier load would have difficulty being approved by the Human Ethics Committee. Dependent on condition, this load was in the form of softner salt packages and weight plates in a hiking backpack, or a child on the back in the position of a piggyback. Because of the fitness and strength levels of these athletes and the relatively low load that was used in this study, the risk was seen as minimal when compared to similar studies (Birrell et al., 2007; Goh et al., 1998).

\section{Testing Protocol}

Testing occurred at the Pan Am Clinic Foundation Biomechanics Laboratory in one session. The passenger was asked to arrive first and participants were scheduled to arrive in 15 minute planned intervals. Participants signed the informed consent form prior to filming, and filled out the Participant Form. Parental consent was obtained for the passenger. The informed consent form was approved by the Education and Nursing review panel of the University of Manitoba. Carriers received one of twelve unlabelled envelopes, half of which stated "NL - BP - PB" and half of which stated "NL - PB - BP" which randomly determined the order of the load conditions. Markers were attached to various locations on the carrier's body, and height and body mass were measured on a scale and recorded. During testing, each participant performed three successful trials of the unloaded condition first, so as to continue the familiarization and warm-up, before performing three trials for each of the two equally-weighted loaded conditions. The passenger tried to maintain a static position throughout each piggyback trial. During each trial, the participant walked naturally across the eight meter walkway with the embedded force plate, while being recorded by video cameras on both the right and left sides (Figure 3). A trial was considered successful if the gait was consistent and natural (Birrell et al., 2007) with the right foot contacting the force plate, and only the force plate (Birrell et al., 2007; Chow et al., 2005; Wannop, Worobets, \& Stefanyshyn, 2012) during the step in question. Each participant performed at least three successful trials of each load condition before filming occurred. During testing, each participant performed three successful trials of the unloaded condition first, so as to continue the familiarization and 


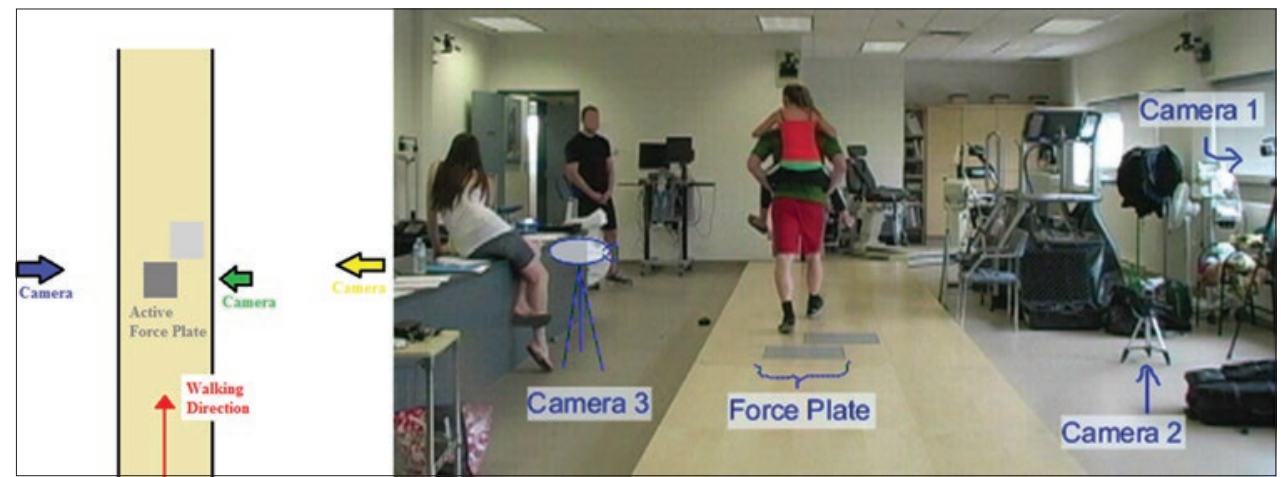

Figure 3. Experimental Set-up. Three cameras filmed the subjects while ground reaction force data was collected by the force plates

warm-up, before performing three trials for each of the two equally-weighted loaded conditions. The passenger tried to maintain a static position throughout each piggyback trial.

The centre of gravity was physically marked on the upper arm and the forearm and hand, while the trunk and head were also marked digitally later in the Dartfish software (Figure 4). In order to identify the location of the centre of gravity of each body segment, the segment endpoints were palpated, the distance between the endpoints was measured, that length was multiplied by the corresponding percentage found in Table 1 , and the resulting distance was measured and marked from the proximal endpoint.

A force plate was used in conjunction with three video cameras and motion analysis software to measure kinematic, gait, and ground reaction force parameters. The embedded Advanced Mechanical Technology, Inc (AMTI) (Advanced Mechanical Technology Inc, 2010) force plate measured the ground reaction forces in three dimensions over time at a frequency of $200 \mathrm{Hertz}$; only the vertical dimension data was used during this analysis. Three cameras filmed the participants at 30 frames per second: two Canon D2L cameras approximately 2.5 meters to either side of the individual as permitted by the laboratory space and one Canon HDV 1080i camera one meter away from the right side of the force plate for a closer view of the feet. The right lateral view was used for all kinematic analysis except in the case of any restrictions, such as when the location of the centre of gravity of the left arm it was hidden behind the trunk. The left lateral view camera was used for such occurrences. All video data was uploaded to the Dell research computer and analyzed using 2014 Dartfish TeamPro 6.0 (Dartfish, 2014).

\section{Data Analysis - Variables Measured}

Kinematic, lumbosacral load, vertical ground reaction force and gait variables were measured for each trial. Each variable was measured at the position determined to be most relevant to biomechanical risk for that specific variable, which is described for each below. For each participant, results from the three successful trials of one condition were averaged to represent that condition (Chow et al., 2005). A key variable measured in this study was maximum trunk flexion angle, but minimum trunk flexion angle and the trunk range of motion were also measured. The maximum trunk flexion angle affects the moments experienced by the lumbosacral spine and
Table 1. Location of the centre of gravity and the relative weight of each relevant body segment by sex - Rearranged from (Hall, 2012) and (Robertson, caldwell, hamill, Kamen, \& Whittlesey, 2004).

\begin{tabular}{lccc}
\hline Segment & \multicolumn{2}{c}{$\begin{array}{c}\text { \% Length from } \\
\text { proximal joint }\end{array}$} & \multirow{2}{*}{$\begin{array}{c}\text { \% Total body } \\
\text { weight * }\end{array}$} \\
\cline { 2 - 3 } & Male & Female $^{*}$ & \\
\hline Head & 55.0 & 55.0 & 7.28 \\
Trunk & 43.6 & 56.9 & 50.70 \\
Upper arm & 43.6 & 45.8 & 2.63 \\
Forearm \& hand & $(62.58)^{*}$ & $(62.58)^{*}$ & 2.27 \\
Thigh & 43.3 & 42.8 & 10.27 \\
Leg & 43.4 & 0.9 & 4.35 \\
Head & 50.0 & 50.0 & 1.47 \\
\hline
\end{tabular}

${ }^{+}$Hall (2012)*Robertson et al. (2004)

is therefore essential to the purpose of the study and relevant with respect to back health. As seen in Figure 5, trunk flexion angle was measured between the vertical axis and the line of the trunk segment using the angle measuring tool in Dartfish. As the video was investigated frame by frame, the moment of least trunk flexion (or maximum extension) was also made a key position and measured in the same way. The difference between these two positions calculated the range of trunk motion. Trunk range of motion was important to monitor; the lumbar spine model uses equilibrium equations that assume that the trunk has a negligible range of motion during gait. The maximum trunk inclination angle for each trial was also used in the lumbar load calculation for that trial.

\section{Kinetic Variables - Lumbosacral Load Variables}

The position of greatest trunk flexion was assumed to be the position of greatest stress on the lumbosacral spine. Therefore, lumbosacral loads were calculated for the instant of maximal trunk flexion using a static biomechanical lumbar spine model. The magnitudes and directions of the unknown resultant muscle moment, the resultant muscle force, and the compressive, shear, and resultant joint reaction forces at the lumbosacral joint were calculated using video position data (Chaffin, Andersson, \& Martin, 1999; McGill, Karpowicz, Fenwick, \& Brown, 2009a; McGill, McDermott, \& Fenwick, 2009b), anthropometrics, and mathematical calculations (Chaffin et al., 1999). 


\section{Lumbosacral Spine Model}

The location of the lumbosacral joint of the carrier was estimated and marked on-screen based on previous research and images of the external view of the body (Moore, Dalley, \& Agur, 2014). The anterior-posterior width of the carrier was measured superior to the iliac crest, slightly superior to the location that would be imagined to present a skin dimple over the posterior superior iliac spine (Khoo, Goh, \& Bose, 1995). The joint was marked at one third of this anterior-posterior distance from the posterior surface of the trunk; this marker represented the mediolateral axis of rotation of the lumbosacral joint.

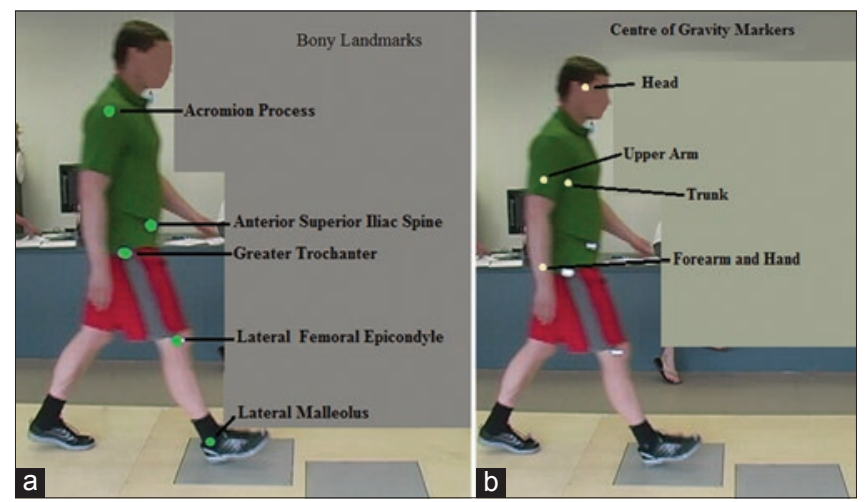

Figure 4. Position Markers. a) Darker tape identified the bony landmarks and b) White tape identified the location of the centre of gravity of important limb segments

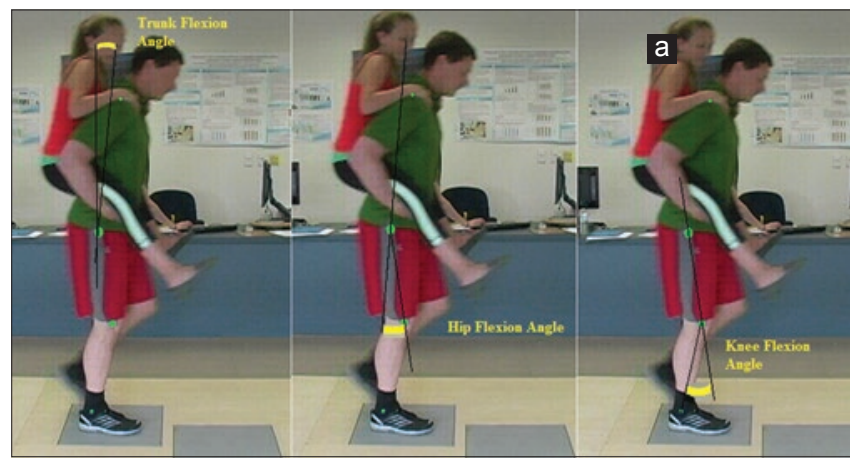

Figure 5. Illustration of trunk, hip, and knee flexion angles

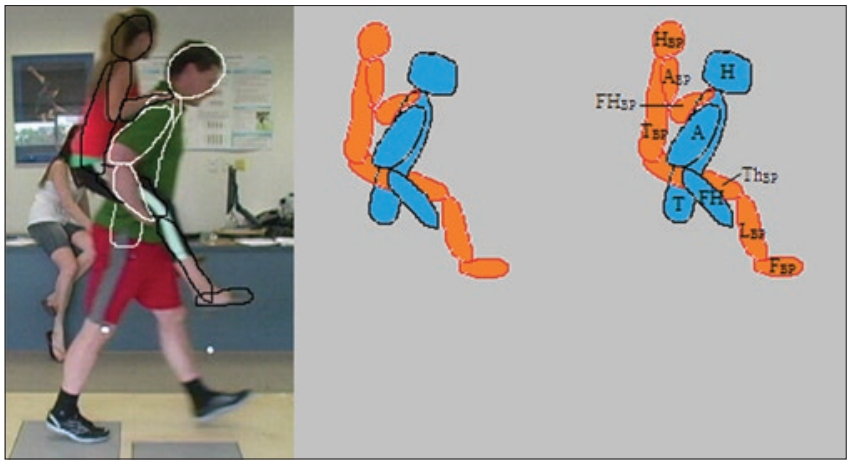

Figure 6. The body segments used for piggyback analysis: the head $(\mathrm{H})$, trunk $(\mathrm{T})$, upper arm $(\mathrm{A})$, and forearm and hand $(\mathrm{FH})$ of the carrier, as well as the head $\left(\mathrm{H}_{\mathrm{BP}}\right)$, trunk $\left(\mathrm{T}_{\mathrm{BP}}\right)$, upper arm $\left(\mathrm{A}_{\mathrm{BP}}\right)$, forearm and hand $\left(\mathrm{FH}_{\mathrm{BP}}\right)$, thigh $\left(\mathrm{Th}_{\mathrm{BP}}\right)$, lower leg $\left(\mathrm{L}_{\mathrm{BP}}\right)$, and foot $\left(\mathrm{F}_{\mathrm{BP}}\right)$ of the person being carried
The body was visually divided into segments connected at joint centres (Figure 6). Only the segments whose weight vectors acted superiorly to the lumbosacral joint centre, creating a moment at the lumbosacral joint, were included. The present model considered four body segments on the carrier and seven body segments on the passenger, each segment represented by its centre of mass location. The head, trunk, upper arm, and lower arm and hand segments were used for both the carrier and passenger (Figure 7). The thigh, lower leg, and foot segments were also used for calculations including the passenger. The force of gravity acting on these segment masses is illustrated in Figure $7 \mathrm{c}$ and is illustrated for backpacking more specifically in Figure 8.

The direction and location of the summed pull of the lumbar spine erector muscles has been estimated and included as one vector representing all lumbar spine extensor muscles, as seen in Figure 9. Although most muscle fascicles do not exert a purely sagittal force (Bogduk, Macintosh, \& Pearcy, 1992) the calculated muscle torque and force will be

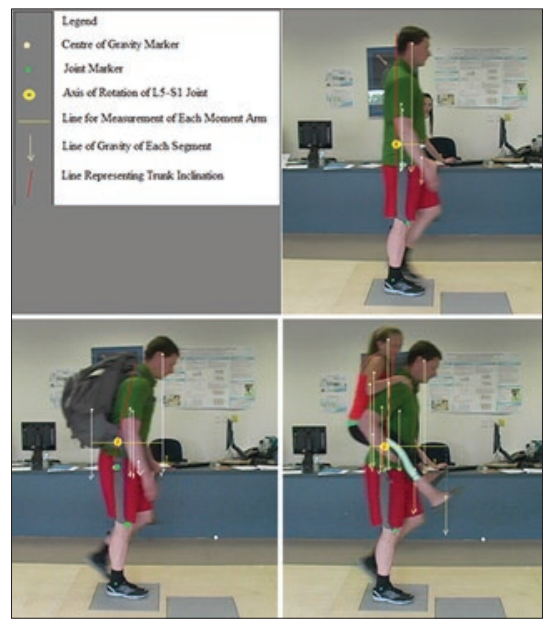

Figure 7. Measurements necessary to calculate lumbosacral load in each frame, over three conditions. The line of force of the muscle has been omitted. A represents the unloaded condition, $\mathrm{B}$ represents the backpack condition, $\mathrm{C}$ represents the piggyback condition

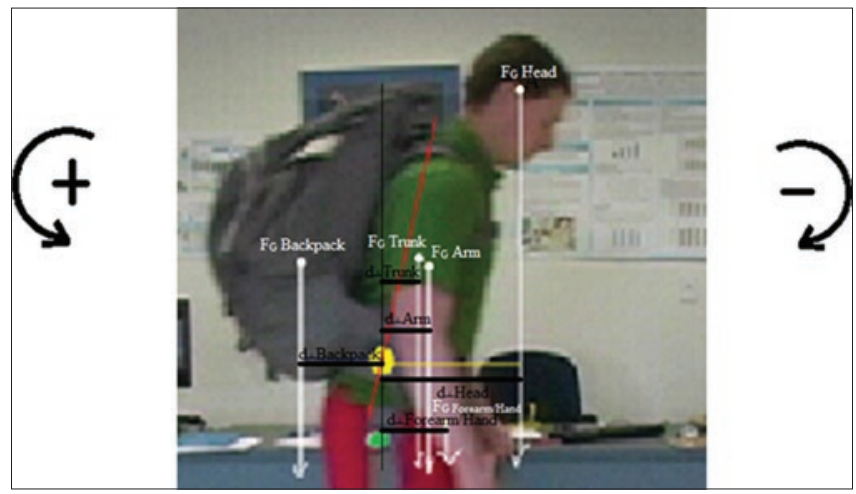

Figure 8. Segment weights and moment arms for a backpack example. The centre of gravity of each segment is represented by a white circle. The line of the force of gravity on each body segment is in white, and each corresponding moment arm is in black. The lumbosacral axis of rotation is located at the yellow dot 


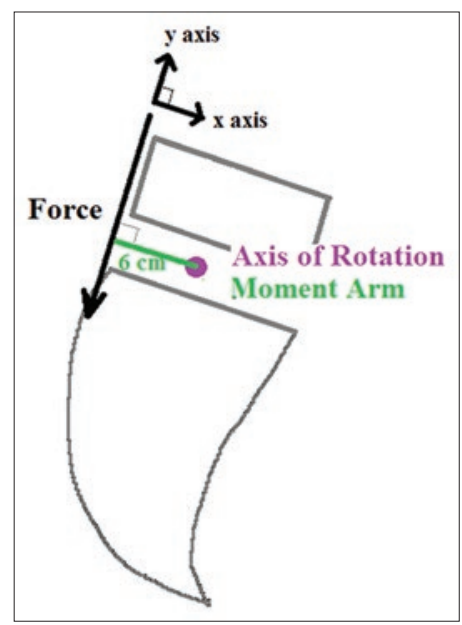

Figure 9. The line of pull and moment arm of the resultant of the summed extensor muscles at the lumbosacral Joint

a reflection of the amount of force that each muscle exerts in only the sagittal plane. With backward rotation representing the positive direction, erector spinae muscle force was considered positive and abdominal muscle force was considered negative. The moment arm of the resultant muscle force was parallel to the surface of the sacrum as it was perpendicular to the line of the muscle force. Based on previous estimations of the length of the resultant extensor muscle moment arm (Cholewicki et al., 1991; Hall, 2012) six centimeters was used in this study.

\section{Calculating Lumbosacral Loads - Muscle Torque and Muscle Force}

Three equations, all based on the assumption of static equilibrium, were used to calculate the lumbar load variables. The first equation balanced the moments about the lumbosacral joint. This provided the muscle torque value, which was divided by the muscle moment arm of six centimeters to produce the muscle force value. The second equation balanced the forces in the $y$ direction to provide the compressive joint reaction force and the third equation added the forces in the $\mathrm{x}$ direction to provide the shear joint reaction force. These equations included the components of the weight of the backpack and each segment of the passenger if they were involved. For example, for the y axis, the sum of the forces perpendicular to the superior sacral surface and parallel to the compressive joint reaction force was equal to zero. These forces were: the compressive joint reaction force, the resultant muscle force, and the y axis components of the weight of each body segment and the backpack.

\section{Kinetic Variables - Vertical Ground Reaction Forces}

The vertical ground reaction forces were graphed over time throughout the duration of right foot stance, and specific peaks were identified. These graphs allowed for the vertical ground reaction force patterns to be visually evaluated. Three specific events of the ground reaction force curve were recorded and compared between each other and between each condition. These were the highest magnitude of the first impact peak, the lowest magnitude of the dip between peaks, and the highest magnitude of the second peak of the vertical ground reaction force.

The ground reaction force values were used both directly and with normalization, which was calculated by dividing the ground reaction force in Newtons by the weight of the entire system in Newtons. The normalized unit was *TW or "times total weight". These three variables were also addressed with respect to each increase from the no load value to the loaded value. Note that both the piggyback load and the backpack load were 284.49 N. For each variable, the no load vGRF was subtracted from the piggyback vGRF. This value was divided by 284.49 and multiplied by 100 to determine the percentage of the added load that manifested as added vGRF. This calculation was repeated for the backpack condition.

A Butterworth filter was tested on the pilot study data, based on recommendations on data filtering (Bogert \& de Koning, 1996). It did not substantially affect the data; for example, it converted one maximum value from $1268.147 \mathrm{~N}$ to 1268.253 N. Due to the results of Bogert (Bogert \& de Koning, 1996) and simple experimentation, the decision was made not to use a filter on the force plate data in this study.

\section{Gait Variables}

Several gait parameters were investigated, including walking velocity, walking cadence, average step length, right to right stride length, left to left stride length, right foot stance time, single stance time, and double stance time (Eddo, Lindsey, Caswell, \& Cortes, 2017). The 0.47 metre force plate width was used as the distance calibration within the plane of the carrier during Dartfish analysis. Walking cadence was also measured and recorded, but in steps per minute. The time it took from left heel strike to left heel strike was measured and inserted into the formula below:

Conversion Formula: (60s) * (2 steps) $\div$ (\# seconds it takes for 2 steps) $=$ Cadence in steps $/$ min

Right to right stride length, and left to left stride length were measured using the distance measuring tool in Dartfish. Right foot stance time was measured from the Excel spreadsheet data collected from the force plate at 200 frames per second. The number of frames in which the right foot was in contact with the ground was counted and multiplied by 0.005 of a second to provide the total amount of time that the right foot was in stance. Single stance time and double stance time were measured onscreen using the times provided by Dartfish video analysis.

\section{Statistical Analysis}

Each variable was compared among the three conditions using a Repeated Measures Analysis of Variance (ANOVA) test utilizing SPSS version 21 software. Mean values were determined for each participant for each variable, and these values were compared amongst the three conditions of no load, piggybacking, and backpacking. An alpha value of 0.05 was used. A normality test was applied to the data to ensure that it was normally distributed. When results were 
significant, a Bonferroni post-hoc test was used to determine which conditions were statistically different from each other.

\section{RESULTS}

\section{Kinematic Variables}

Results for all kinematic variables are listed in Table 2. Every variable exhibited significant differences between at least two conditions.

The maximum angle of trunk flexion while walking was different between all three conditions (Figure 10), based on a repeated measures ANOVA with a Bonferroni post hoc test (Figure 11).

Trunk inclination increased from no load to piggybacking $(p=0.000)$, from no load to backpacking $(p=0.000)$, and from piggybacking to backpacking $(\mathrm{p}=0.002)$ as demonstrated visually in Figure 10 and graphically in Figure 11. The trunk was most vertical when carrying no load $\left(0.9 \pm 1.1^{\circ}\right)$, flexed when piggybacking a person $\left(9.7 \pm 3.1^{\circ}\right)$, and the most flexed when carrying an equally-weighted backpack $\left(12.4 \pm 2.3^{\circ}\right)$.

The angle of relative maximum hip flexion increased from no load to each of the loaded conditions. Because hip flexion angle was measured between the line extended from the trunk and the line of the thigh, this angle reflected the change in trunk inclination. When the influence of trunk inclination on hip flexion angle was investigated by subtracting maximal trunk flexion angle from maximal hip flexion angle, the average absolute hip flexion angles from the vertical axis were approximately $27 \pm 3^{\circ}, 26 \pm 3^{\circ}$ and $26 \pm 3^{\circ}$ for no load, piggybacking and backpacking respectively. Load condition did not determine absolute hip flexion angle, and the previous differences in relative hip angle were likely only a result of trunk flexion.

The angle of maximum knee flexion was greatest when carrying a backpack, which varied from maximum knee flexion angle when carrying a person and when carrying no load. The mean maximum knee angle for piggybacking was also greater than the mean for no load, but this difference was not significant.

\section{Torque}

Every external lumbosacral torque variable was different between all three conditions except for the total amount of negative torque, which was similar between the two loaded conditions. Details of the load torque variables estimated by the lumbar spine model are included in Table 3. Both the resultant torque and total magnitude of the torque caused by the load were statistically greater for the backpack than for the passenger. For the piggyback condition, the torques produced by the body segments of the passenger were divided into positive and negative torques. Approximately $25 \%(-8.45 \pm 1.51 \mathrm{~N})$ of the total magnitude of torque caused by the passenger was negative, while approximately $75 \%(25.13 \pm 3.88 \mathrm{~N})$ of the passenger torque was positive. Overall, the load itself increased the tendency to rotate backward during piggybacking.

Table 2. Kinematic variable means, standard deviations, and pairwise p-values across conditions

\begin{tabular}{lcccccc}
\hline Variablelcondition & No load & Piggyback & Backpack & \multicolumn{3}{c}{ Bonferroni p-value } \\
\cline { 4 - 7 } & & & & NL/BP & NL/PB & BP/PB \\
\hline Max trunk flex angle $\left(^{\circ}\right)$ & $0.9 \pm 1.1$ & $9.7 \pm 3.1$ & $12.4 \pm 2.3$ & 0.000 & 0.000 & 0.002 \\
Min trunk flex angle $\left(^{\circ}\right)$ & $-0.1 \pm 1.3$ & $7.3 \pm 3.2$ & $8.2 \pm 3.2$ & 0.000 & 0.000 & 0.927 \\
Trunk flex-ext ROM $\left(^{\circ}\right)$ & $2.2 \pm 1.1$ & $2.3 \pm 1.1$ & $4.2 \pm 1.7$ & 0.002 & 1.000 & 0.003 \\
Max hip flex angle $\left(^{\circ}\right)$ & $27.5 \pm 2.9$ & $35.5 \pm 3.3$ & $38.4 \pm 3.5$ & 0.000 & 0.000 & 0.050 \\
Max knee flex angle $\left(^{\circ}\right)$ & $17.8 \pm 8.9$ & $22.6 \pm 3.0$ & $24.2 \pm 3.7$ & 0.026 & 0.126 & 0.026 \\
\hline
\end{tabular}

a A Bonferroni post hoc test was used if repeated measures ANOVA determined significance, Statistically significant $\mathrm{p}$-values are bolded, $\alpha=0.05$

Table 3. Lumbosacral torque means, standard deviations, and pairwise p-values

\begin{tabular}{|c|c|c|c|c|c|c|}
\hline \multirow[t]{2}{*}{ Variablelcondition } & \multicolumn{2}{|c|}{ Torque (T) (Nm) } & \multirow[t]{2}{*}{ Backpack } & \multicolumn{3}{|c|}{ Bonferroni p-value } \\
\hline & No load & Piggyback & & NL/BP & NL/PB & BP/PB \\
\hline Resultant torque ( $\mathrm{T}$ ) by load only & & $16.68 \pm 4.81$ & $54.69 \pm 6.67$ & & & 0.000 \\
\hline Total magnitude of $\mathrm{T}$ by load only & & $33.42 \pm 3.33$ & $54.69 \pm 6.67$ & & & 0.000 \\
\hline $\mathrm{T}$ by body of carrier & $-27.66 \pm 6.42$ & $-38.70 \pm 7.27$ & $-48.73 \pm 8.75$ & 0.000 & 0.000 & 0.000 \\
\hline Total resultant $\mathrm{T}$ & $-27.66 \pm 6.42$ & $-22.01 \pm 10.73$ & $5.96 \pm 13.15$ & 0.045 & 0.000 & 0.000 \\
\hline Total magnitude of $\mathrm{T}$ & $27.66 \pm 6.42$ & $72.28 \pm 5.58$ & $103.41 \pm 8.33$ & 0.000 & 0.000 & 0.000 \\
\hline Total negative $\mathrm{T}$ & $-27.66 \pm 6.42$ & $-47.15 \pm 7.62$ & $-48.73 \pm 8.75$ & 0.000 & 0.000 & 0.760 \\
\hline Total negative $\mathrm{T}$ as $\%$ & $100.00 \pm 0.00$ & $65.17 \pm 6.53$ & $46.92 \pm 6.57$ & 0.000 & 0.000 & 0.000 \\
\hline Total positive $T$ & $0.00 \pm 0.00$ & $25.14 \pm 3.88$ & $54.69 \pm 6.67$ & 0.000 & 0.000 & 0.000 \\
\hline Total positive $\mathrm{T}$ as $\%$ & $0.00 \pm 0.00$ & $34.83 \pm 6.53$ & $53.08 \pm 6.57$ & 0.000 & 0.000 & 0.000 \\
\hline
\end{tabular}

A Bonferroni post hoc test was used when the repeated measures ANOVA determined significance, Instead, a Paired T-Test was used when only two conditions were compared, Statistically significant $p$-values are bolded, $\alpha=0.05-$, Backward rotation was considered positive 


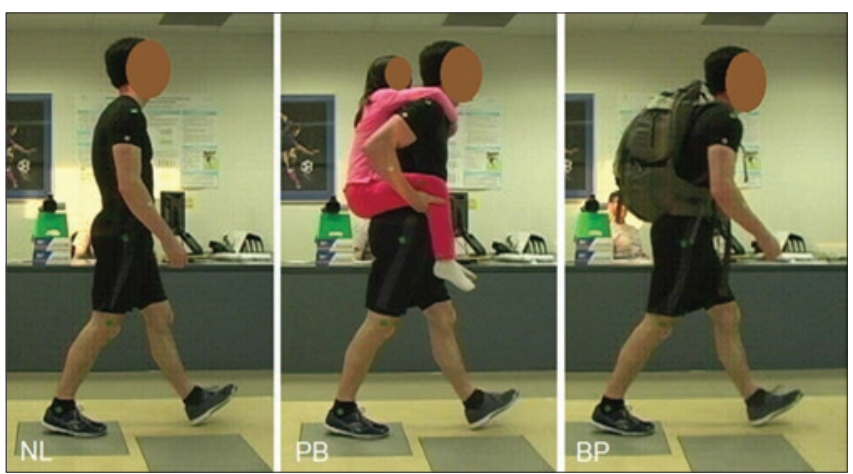

Figure 10. Still frames at maximum trunk flexion by load condition

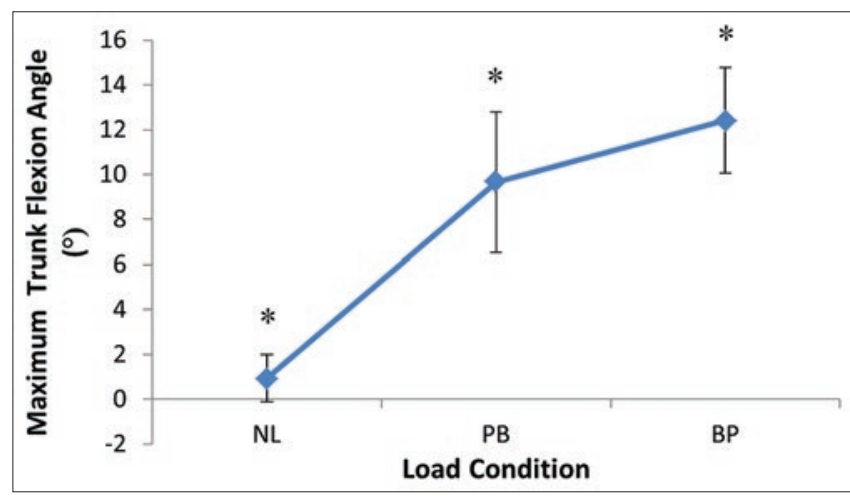

Figure 11. Maximum trunk flexion angle by load condition. *At $\alpha=0.05$, the maximum angle of trunk flexion was significantly different between each pair of load conditions

The total resultant torque increased from no load to the piggyback condition to the backpack condition; both the no load and piggyback conditions demonstrated an overall tendency for the system to rotate forward while the backpack condition demonstrated a tendency for the system to rotate backward. For piggybacking, the anterior body segments of both the carrier and passenger created more negative torque than the portion of the passenger that produced postive torque. The positive torque produced by the backpack was close to but greater than the negative torque produced by the body segments of the carrier. When summed, the resultant torque described the overall tendency for rotation around the medio-lateral axis of the lumbosacral joint.

\section{Lumbar Loads}

All estimated lumbar loads are listed in Table 4. The torque calculation relies on the balancing of torques, which only provides the resultant torque in one direction rather than the absolute torque in each direction. Because resultant torque magnitude must be less than absolute torque magnitude and the remaining lumbar load variables were calculated based on the resultant muscle torque, these variables were underestimations to some degree. Resultant muscle torque increased from the backpack condition to the piggyback condition to the no load condition. The resultant muscle torque was a negative value in the backpack condition and a positive value in the other two conditions; therefore, the trunk extensors produced greater torque than the trunk flexors during the piggybacking and no load conditions, but the trunk flexors dominated during backpacking. The resultant muscle torque was very similar between piggybacking and carrying no load, with a Pearson correlation coefficient of 0.8 .

The resultant muscle force averages, seen in Table 4, were statistically different between all pairs of conditions, increasing in magnitude from backpacking to piggybacking to no load. The backpack condition had a negative value and the other two conditions had a positive value; the trunk extensors dominated during backpacking and the trunk flexors dominated during both piggybacking and unloaded walking.

The estimated compressive, shear and resultant joint reaction force values are illustrated in Figure 12 for comparison. The compressive and resultant joint reaction force values were both statistically different between each of the three conditions, increasing from no load to piggybacking to backpacking. The estimated shear joint reaction force value increased from no load to each of the identical load condition values. This equality was a direct and predictable result of the lumbar spine model; the shear calculation depended on total load, for which the loaded conditions had identical values.

\section{Vertical Ground Reaction Force Variables}

Walking over a force plate usually generates a predictable butterfly pattern (Robertson et al., 2004). The curves for each trial were slightly different from each other: some with smoother and less variable lines than others, some first peaks greater than the second peaks, and some the opposite. The mean first peak value was less than the mean second peak value when walking unloaded and when backpacking, whereas the peaks were almost equal when piggybacking. The curves tended to be more similar within participants and across conditions than between participants, suggesting that each individual has a specific way of moving that they will repeat when they are asked to repeat an activity.

Relative vertical ground reaction force variable results are reported in Table 5 and are illustrated in Figure 13. The first peak was different between piggybacking and backpacking, the interpeak minimum exhibited no differences, and the second peak was different between no load and piggybacking. For all conditions, the mean first vGRF peak was measured at slightly greater than body weight, increasing from backpacking to no load to piggybacking. All interpeak minimums were approximately three quarters of body weight. The second vGRF peak was greatest for the unloaded condition, less for the backpack condition, and the least for the piggyback condition. Participants exerted a greater second relative vertical ground reaction force peak when carrying no load as compared to piggybacking. Therefore, the participants exerted a smaller downward force during the propulsive phase of piggybacking than during the propulsive phase of the no load condition.

Absolute vertical ground reaction force variable results are listed in Table 6. The no load condition produced a lower vGRF than each loaded condition during the first peak, the 
Table 4. Lumbar load means, standard deviations, and pairwise p-values across conditions

\begin{tabular}{|c|c|c|c|c|c|c|}
\hline \multirow[t]{2}{*}{ Variablelcondition } & \multirow[t]{2}{*}{ No load } & \multirow[t]{2}{*}{ Piggyback } & \multirow[t]{2}{*}{ Backpack } & \multicolumn{3}{|c|}{ Bonferroni p-value } \\
\hline & & & & NL/BP & NL/PB & $\mathbf{B P} / \mathbf{P B}$ \\
\hline Resultant muscle torque $(\mathrm{Nm})$ & $27.66 \pm 6.42$ & $21.77 \pm 10.91$ & $-5.96 \pm 13.15$ & 0.000 & 0.039 & 0.000 \\
\hline Resultant muscle force $(\mathrm{N})$ & $460.98 \pm 107.08$ & $362.80 \pm 181.80$ & $-99.40 \pm 219.39$ & 0.000 & 0.039 & 0.000 \\
\hline Compressive L5-S1 JRF (N) & $14.49 \pm 79.57$ & $355.40 \pm 150.27$ & $821.62 \pm 181.02$ & 0.000 & 0.000 & 0.000 \\
\hline Shear L5-S1 JRF (N) & $272.24 \pm 27.43$ & $413.59 \pm 27.43$ & $413.59 \pm 27.43$ & 0.000 & 0.000 & 1.000 \\
\hline Resultant L5-S1 JRF (N) & $287.08 \pm 30.50$ & $581.84 \pm 82.97$ & $923.50 \pm 155.22$ & 0.000 & 0.000 & 0.000 \\
\hline
\end{tabular}

A Bonferroni post hoc test was used when the repeated measures ANOVA determined significance, Statistically, significant p-values are bolded, $\alpha=0.05$

Table 5. Relative vertical GRF variable means and standard deviations by total weight $(* T W)$, with pairwise p-values across conditions

\begin{tabular}{lcccccc}
\hline Variablelcondition & No load & Piggyback & Backpack & \multicolumn{3}{c}{ Bonferroni p-value } \\
\cline { 5 - 7 } & & & & NL/BP & NL/PB & BP/PB \\
\hline $1^{\text {st }}$ Peak & $1.08 \pm 0.09$ & $1.09 \pm 0.06$ & $1.05 \pm 0.06$ & 0.305 & 1.000 & 0.011 \\
Interpeak minimum $(* \mathrm{TW})$ & $0.75 \pm 0.06$ & $0.72 \pm 0.06$ & $0.72 \pm 0.08$ & 0.073 & 0.102 & 1.000 \\
$2^{\text {nd }}$ Peak $(* \mathrm{TW})$ & $1.12 \pm 0.05$ & $1.06 \pm 0.04$ & $1.09 \pm 0.05$ & 0.095 & 0.001 & 0.119 \\
\hline
\end{tabular}

A Bonferroni post hoc test was used when the repeated measures ANOVA determined significance, Statistically significant p-values are bolded, $\alpha=0.05$

Table 6. Absolute vertical ground reaction force variable means, standard deviations, and pairwise p-values across conditions

\begin{tabular}{|c|c|c|c|c|c|c|}
\hline \multirow[t]{2}{*}{ Variable $\backslash$ condition } & \multirow[t]{2}{*}{ No Load } & \multirow[t]{2}{*}{ Piggyback } & \multirow[t]{2}{*}{ Backpack } & \multicolumn{3}{|c|}{ Bonferroni p-value } \\
\hline & & & & NL/BP & NL/PB & BP/PB \\
\hline $1^{\text {st }}$ Peak & $875.75 \pm 107.85$ & $1196.62 \pm 106.04$ & $1059.84 \pm 345.70$ & 0.000 & 0.000 & 0.013 \\
\hline Interpeak minimum $(\mathrm{N})$ & $608.74 \pm 82.52$ & $790.06 \pm 86.61$ & $788.50 \pm 107.84$ & 0.000 & 0.000 & 1.000 \\
\hline $2^{\text {nd }}$ Peak $(\mathrm{N})$ & $904.99 \pm 94.73$ & $1161.98 \pm 100.58$ & $1186.64 \pm 99.33$ & 0.000 & 0.000 & 0.109 \\
\hline
\end{tabular}

A Bonferroni post hoc test was used if the repeated measures ANOVA determined significance, Statistically significant $p$-values are bolded, $\alpha=0.05$

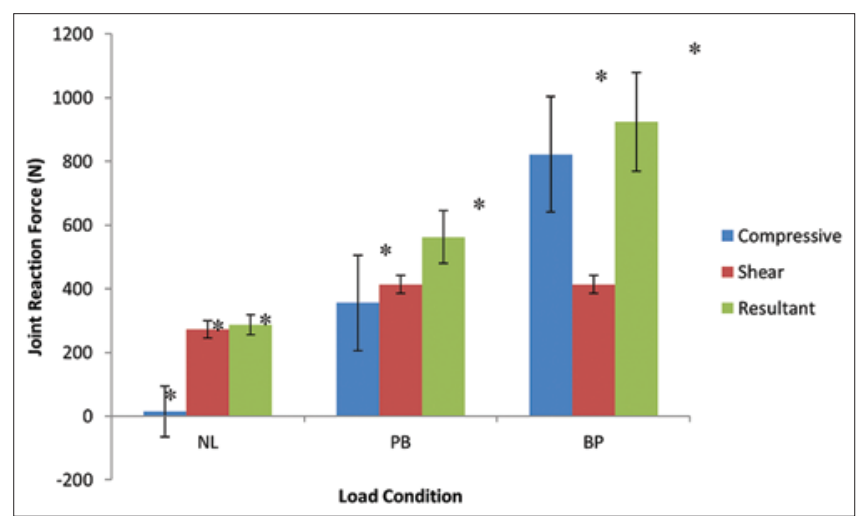

Figure 12. Estimated shear, compressive, and resultant joint reaction forces by load condition. * At $\alpha=0.05$, the compressive and resultant joint reaction forces were different between all load conditions; the shear joint reaction force was less for the no load condition than for the loaded conditions

interpeak minimum, and the second peak of the vGRF curve. The only difference between the loaded conditions was that the first absolute vGRF peak was less for piggybacking than backpacking (Figure 13).

\section{DISCUSSION}

The addition of a load primarily posterior to the trunk caused increased trunk flexion, a finding supported by several previous studies (Gillet et al., 2006; Goh et al., 1998; Kinoshita, 1985; Rodriguez-Soto et al., 2013; Vacheron, Poumarat, Chandezon, \& Vanneuville, 1999). This flexion also increased between equal loads from piggybacking to backpacking. The mean trunk flexion angle values were $0.9 \pm 1.1^{\circ}, 9.7 \pm 3.1^{\circ}$, and $12.4 \pm 2.3^{\circ}$ for walking with no load, piggybacking, and backpacking, increasing from no load by approximately $11.5^{\circ}$ with the addition of the $29 \mathrm{~kg}$ backpack. Previously, the addition of a $15 \mathrm{~kg}$ backpack increased trunk flexion during gait from $4.9 \pm 2.7^{\circ}$ to $14.2 \pm 3.2$. That load was lighter than the current $29 \mathrm{~kg}$ load, and the trunk flexion increase was slightly less. When $3 \mathrm{~kg}$ of that load was shifted anteriorly, similar to piggybacking, the trunk angle was $10.6 \pm 2.9^{\circ}$ (Gillet et al., 2006). This front-back pack trunk flexion angle was between the angles for no load and fully posterior conditions, similar to the current piggyback values. Other researchers measured a $25^{\circ}$ to $34^{\circ}$ increase in trunk flexion with a $58.8 \mathrm{~kg}$ backpack and body armour load (Rodriguez-Soto et al., 2013); this greater load demonstrated 


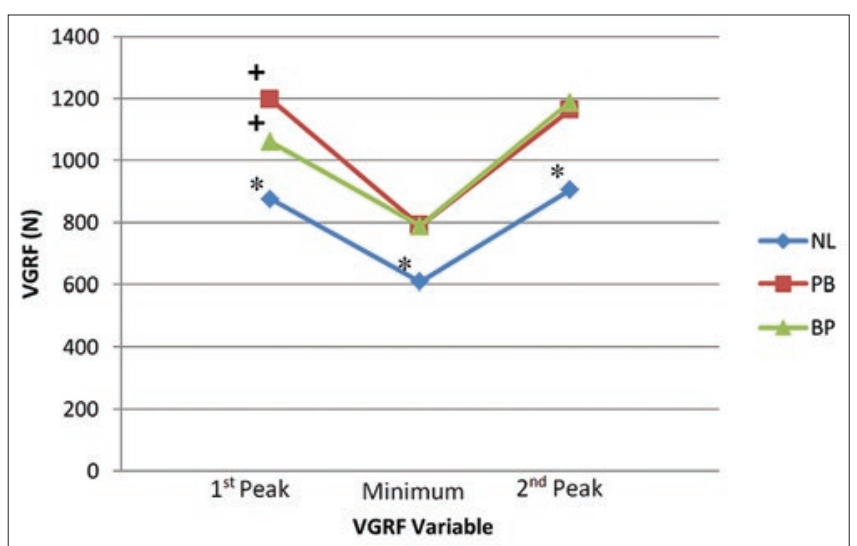

Figure 13. The absolute $1^{\text {st }}$ peak, interpeak minimum, and $2^{\text {nd }}$ peak of the vertical ground reaction force curves for each load condition, illustrating the typical butterfly patterns. *At $\alpha=0.05$, the no load condition was less than each of the two loaded conditions for every absolute vGRF variable. The first vGRF peak value was also less for piggybacking compared to backpacking $(+)$
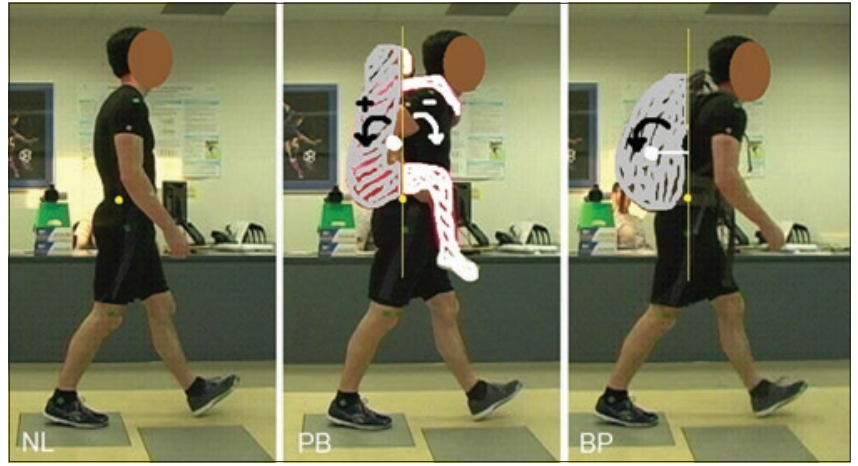

Figure 14. A comparison of positive and negative torques produced by the load and the position of the overall centre of gravity of the load with corresponding moment arms. Backwardrotating torques are represented by black arrows and forwardrotating torques are represented by a white arrow. The centre of gravity of each load is represented by a large white dot. The moment arm of each load is represented by a white horizontal line connecting its centre of gravity to the vertical line at the lumbosacral joint

greater trunk flexion than in the present study, which is reasonable. Variations between study results also occur due to the different subjects being tested, data collection methods, and body landmark locations used.

The original hypothesis for the cause of trunk flexion with a posterior load was supported by Goh et al. (Goh et al., 1998) who first articulated the term "compensatory trunk flexion". This term (Goh et al., 1998) suggested that participants instinctively shifted their body segments forward to create forward torque to counteract the backwards torque created by an added posterior load. Gillet et al. (Gillet et al., 2006) suggested that the backwards rotating moment caused by the posterior load is compensated for by an increase of six to seven degrees of trunk flexion. The loads added posterior to the trunk shifted the centre of gravity of the system posteriorly, creating an extensor torque which would likely cause the participant to fall backward if not for the reactive trunk flexion that was exhibited (Goh et al., 1998). The further posterior the centre of gravity or the greater the mass of the load, the greater the posterior shift in the centre of gravity of the system, and the greater the trunk flexion to be expected. The trunk flexes to shift the centre of gravity anteriorly so that it is over the base of support (McGill, Marshall, \& Andersen, 2013).

The conclusion that piggybacking allows for a more natural gait than backpacking is not only supported by the results of several variables in the present study, but also by load carriage studies that involved front-back packs (Gillet et al., 2006; Kinoshita, 1985; Legg, 1985). The front portion or pocket of a front-back pack is comparable to the arms and legs of a piggyback passenger, as they wrap around to the front of the body. Therefore, after producing the results of the current study, the conclusion remains that front-back packs are the closest representative of piggybacking in existing literature, and that they exhibit less deviation from normal gait when compared to backpacking.

The angle of maximal knee flexion increased from no load $\left(17.8 \pm 8.9^{\circ}\right)$ to backpacking $\left(24.2 \pm 3.7^{\circ}\right)$ and from piggybacking $\left(22.6 \pm 3.0^{\circ}\right)$ to backpacking. With the addition of a load, the carriers may have subconsciously altered gait to lessen the increased impact with the ground (Chow et al., 2005). This "cushioning" could be performed by any combination of joints in the sagittal plane. Because the absolute hip angle did not alter and the knee flexion angle did increase when backpacking, it is likely that this cushioning was primarily performed by the knee joint. Because there was an additional $29 \mathrm{~kg}$ of mass to be controlled and absorbed at impact with the ground, the piggybacking knee flexion angle would be expected to be greater than the no load knee flexion angle even if other gait mechanics were identical. The knee flexion results support the theory that piggybacking is biomechanically more similar to natural gait than is backpacking. The resultant torques caused by the passenger and backpack were both positive or backward, but the passenger $(16.68 \pm 4.81 \mathrm{Nm})$ caused less backward torque than the backpack $(54.69 \pm 6.67 \mathrm{Nm})$ because of their different positions and resulting moment arms (Figure 14). The moment arm from the centre of gravity of the backpack to the lumbosacral joint was longer than the sum of the moment arms from the centre of gravity of each passenger segment to the lumbosacral joint; the weight of the backpack load acted at a greater distance from the axis of rotation than did the overall weight of the passenger load. This longer moment arm for an equal load resulted in a greater positive torque produced by the backpack than the passenger (Hall, 2012).

When the magnitudes of the positive and negative portions of the load torque were summed to the total magnitude of load torque, the passenger $(33.42 \pm 3.33 \mathrm{Nm})$ caused less total magnitude of torque than the backpack $(54.69 \pm 6.67$ $\mathrm{Nm}$ ) even though the weights were equal. This was due to the longer moment arm of the backpack weight in comparison to the sum of the moment arms for the passenger segment weights as described above. The body segments of the passenger tended to be closer to the lumbosacral joint than the backpack, with some in positive and some in negative directions. The segments that were further from the joint and 
were in the position to create larger torques only had small masses such as the $8.36 \mathrm{~N}$ feet. The greater magnitude of torque produced by the backpack suggests that backpacking is generally more strenuous than piggybacking. The backpack created a backward torque that was almost balanced by the upper body segments of the carrier; the backpack only produced approximately $6 \mathrm{Nm}$ more torque than did the upper body segments. This suggested that the trunk instinctively flexed forward to a fairly balanced position in response to the backpack load, possibly to ease some of the muscle work. These torques can be placed into context by considering that the L4-L5 moments were $61 \mathrm{Nm}$ to $267 \mathrm{Nm}$ when walking and carrying heavy loads (McGill et al., 2009b) over $200 \mathrm{Nm}$ for other lifting tasks (McGill \& Norman, 1985) and over $900 \mathrm{Nm}$ for extreme lifting (Cholewicki et al., 1991). Considering the much less extreme position of load carriage demonstrated in this study, it is reasonable that the torque value was much less. A summary of the torques acting during each condition, based on the averages of various variables, is included in Figure 15. Included variables are the trunk flexion angle, total resultant torque, total negative torque, negative torque as a percentage of total magnitude of torque, total positive torque, positive torque as a percentage of total magnitude of torque, and resultant muscle torque. Figure 165 illustrates substantial differences in the torques experienced between these two equally-loaded conditions. Note the similar amounts of positive torque but substantially greater negative torque during backpacking. In both cases, the positive torque was caused by the upper body of the carrier, with the addition of part of the passenger for the piggyback condition. The entire backpack caused negative torque while only the remaining portions of the passenger caused negative torque, and with a lesser moment arm.

Overall, this diagram illustrates that with respect to trunk flexion and torque, the piggyback condition was much more similar to unloaded walking than was the backpack condition. In fact, the no load and piggyback conditions were found to have opposite trunk muscle forces dominating as compared to the backpack condition. The resultant muscle torque increased from backpacking $(-5.96 \pm 13.15 \mathrm{Nm})$ to piggybacking $(21.77 \pm 10.91 \mathrm{Nm})$ to no load $(27.66 \pm 6.42 \mathrm{Nm})$;

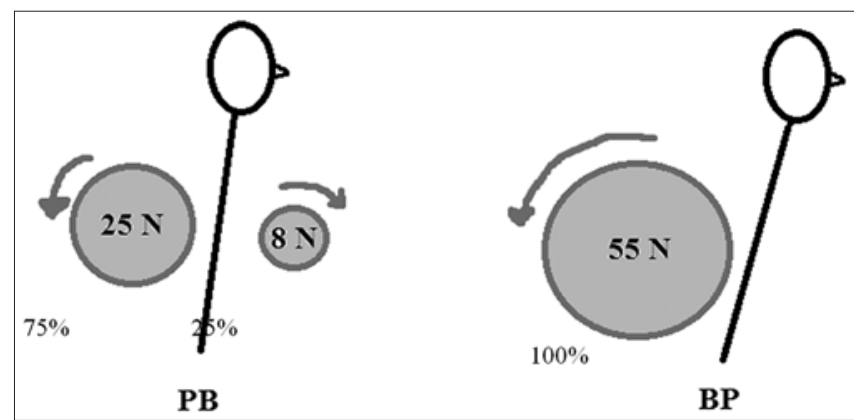

Figure 15. A comparison of the forward and backward mediolateral lumbosacral joint torques produced during piggybacking and backpacking by the $29 \mathrm{~kg}$ load alone. The positive and negative magnitudes are subtracted to determine resultant load torque and added to determine total magnitude of load torque the overall muscle pull rotated the trunk forward during backpacking (trunk flexors) and backward during piggybacking and when unloaded (trunk extensors). This muscle torque was a direct response to counteract the total resultant torque experienced in the opposite direction, in order to maintain trunk position. The vertical ground reaction force patterns demonstrated the typical butterfly pattern with two major peaks and one interpeak dip (Robertson et al., 2004) during all three conditions. The vGRF is known to stay close to body weight, fluctuating by approximately $30 \%$ of body weight (Robertson et al., 2004). The first peak was expected to be around 1.1 times body weight. The relative vGRF interpeak minimum was approximately three quarters of the total weight. This is typical based on previous values, such as just under 0.8 to 0.85 (Robertson et al., 2004). The relatively small range of vertical forces between the first peak and interpeak dip suggests that the participants had minimal vertical accelerations, which corresponded visually to the video footage.

The second vGRF peak increased from the piggybacking $(1.06 \pm 0.04 * \mathrm{TW})$ to the no load condition $(1.12 \pm 0.05$ *TW). Typical values are approximately 1.06 to 1.2 times body weight at the second peak (Robertson et al., 2004). This difference illustrated that although the gait pattern did not alter much by condition, the participants felt more comfortable to push off of the ground more forcefully during the propulsive phase of the unloaded condition than for the piggyback condition. This is logical because an individual carrying a backpack or person may be concerned with the risk of dropping his bag or passenger, when an unloaded individual would only be concerned about himself and he would be accustomed to accelerating his own body. It may also be that rather than being concerned about their load, this difference was caused by gait alterations that made it easier for the participants to control the load.

Contrary to expectations, the only two gait variables that demonstrated differences between conditions were right foot stance time and double stance time. If carrying load above the head is considered similar to piggybacking because the centre of gravity of the load is better aligned with the centre of gravity of the carrier, then these findings are also supported by the results of Lloyd et al. (Lloyd, Parr, Davies, \& Cooke, 2011). Stance time increased from carrying no load to carrying the load superior to the head to carrying a load posterior to the spine (Lloyd et al., 2011).

Right foot stance time was seen to increase when measured at $200 \mathrm{~Hz}$ so it is assumed that left foot stance time would have also increased, and these increases would have led to an increase in double stance time. If this assumption is true despite the lack of significant data, the $30 \mathrm{fps}$ video data likely was not sufficiently frequent to identify these changes. However, double stance time did increase from no load to backpacking, which further suggests the need for longer ground contact time while carrying a backpack (Birrell et al., 2007; Chow et al., 2005; Cottalorda et al., 2003).

This study was limited by the weight of the subject being carried piggyback which was only $29 \mathrm{~kg}$, or forty per cent of the weight of the athlete carrying her. When training 
for Crossfit the athlete would be carrying a much heavier weight, as often the subject being carried is heavier than the carrier. The University Ethics Board does not allow research subjects to carry loads that are potentially unsafe. The study implies that it is less stressful on the lumbar spine if the load is carried by a piggyback carry rather than a backpack carry or other solid weight. The piggyback carry allows some adjustment by the passenger to move the body segments closer to the center of gravity of the carrier, decreasing the torques produced by each segment.

\section{CONCLUSIONS}

The findings suggest that in CrossFit training and competitions, for any given weight the piggyback carry may be safer than some of the other training activities in which the load is carried either in the arms in front of the body or loaded onto the back. The conclusion that piggybacking allows for a more natural gait than backpacking or other weight carries is supported by the results of several variables in the present study. Any carry in which the person being carried moves their body parts closer to the center of gravity or to the lumbo-sacral axis will decrease torques about these axes and decrease the compressive and shear forces acting across the body of the sacrum. The participant should also try to limit the amount of trunk flexion occurring during these weightlifting exercises, as this adjustment may also decrease the magnitude of the resistance torques being lifted by decreasing the magnitude of the moment arms produced. Injury can potentially occur when the athlete attempts to carry a heavy weight located too far in front of or behind the lumbosacral axis, maximizing the resultant joint torques and joint reaction forces to be overcome.

\section{REFERENCES}

Advanced Mechanical Technology Inc. (2010). AMTI Force and Motion. Retrieved from http://amti.biz/index.aspx

Beekley, M. (2007). Effects of heavy load carriage during constant-speed, simulated, road marching. Military Medicine, 172(6), 592-595

Bennett, G. (1999, may 25, 1999.). Lessons in life- from rough-housing: wrestling, tickling and piggyback rides are activities full of life lessons for boys and girls., The Ottawa Citizen, p. A14.

Birrell, S., Hooper, R., \& Haslam, R. (2007). The effect of military load carriage on ground reaction forces. Gait \& Posture, 26(4), 611-614

Bogduk, N., Macintosh, J. E., \& Pearcy, M. J. (1992). A universal model of the lumbar back muscles in the upright position. Spine, 17(8), 897-913

Bogert, A. J., \& de Koning, J. J. (1996). On optimal filtering for inverse dynamics analysis. In A. Hoffer (Ed.), Canadian Society for Biomechanics Ninth (pp. 214-215). Vancouver, B.C,: Canadian Society for Biomechanics.

Chaffin, D. B., Andersson, G. B. J., \& Martin, B. J. (1999). Occupational Biomechanics $\left(3^{\text {rd }}\right.$ ed.). New York, NY: John Wiley \& Sons, Inc.
Cholewicki, J., McGill, S. M., \& Norman, R. W. (1991). Lumbar spine loads during the lifting of extremely heavy weights. Medicine and Science in Sports and Exercise, 23(10), 1179-1186

Chow, D., Kwok, M., Au-Yang, A., Holmes, A., Cheng, J., Yao, F., \& Wong, M. (2005). The effect of backpack load on the gait of normal adolescent girls. Ergonomics, 48(6), 642-656

Cottalorda, J., Rahmani, A., Diop, M., Gautheron, V., Ebermeyer, E., \& Belli, A. (2003). Influence of school bag carrying on gait kinetics. Journal of Pediatric Orthopaedics $B, 112(6), 357-364$

Crossfit Inc. (2008). CrossFit: Forging Elite Fitness. Retrieved from http://board.crossfit.com/showthread. php? $\mathrm{t}=25759$ \&page $=25$

Crossfit Winnipeg. (2014). Crossfit Winnnipeg Health, Fitness and Performance. Retrieved from http://www. crossfitwinnipeg.com

Dartfish. (2014). Dartfish Digital Video Analysis System. Retrieved from http://www.dartfish.com

Donohue, D. P. (1996, Feb 3, 1996). Smoking cancels benefits of aerobic exercise, Edmonton Journal, p. C8.

Eddo, O., Lindsey, B., Caswell, S. V., \& Cortes, N. (2017). Current Evidence of Gait Modification with Real-time Biofeedback to Alter Kinetic, Temporospatial, and Function-Related Outcomes: A Review. International Journal of Kinesiology \& Sports Science, 5(3), 35-55

Faul, F., Erdfelder, E., Lang, A. G., \& Buchner, A. (2007). G*Power 3: A flexible statistical power analysis program for the social, behavioral, and biomedical sciences. Behavior Research Methods, 39, 175-191

Gillet, C., Foissac, M., Leteneur, S., Freychat, P., \& Babier, F. (2006). Influence of a new backpack design on kinematics and dynamics of walking. The Engineering of Sport, 6(3), 369-374

Goh, J. H., Thambyah, A., \& Bose, K. (1998). Effects of varying backpack loads on peak forces in the lumbosacral spine during walking. Clinical Biomechanics, 13(1), S26-S31

Grenier, J. G., Millet, G. Y., Peyrot, N., Salmozino, P., Oullion, R., Messonnier, L., \& Morin, J. (2012). Effects of extreme-duration heavy load carriage on neuromuscular function and locomotion: a military-based study. PLOS One, 7(8), 1-11

Hall, S. J. (2012). Basic Biomechanics (6 $6^{\text {th }}$ Edition). New York, NY: McGraw-Hill Higher Education.

Khoo, B. C., Goh, J. C., \& Bose, K. (1995). A biomechanical model to determine lumbosacral loads during single stance phase in normal gait. Medical Engineering Physics, 17(1), 27-35

Kinoshita, H. (1985). Effects of different loads and carrying systems on selected biomechanical parameters describing walking gait. Ergonomics, 28(9), 1347-1362

Legg, S. J. (1985). Comparison of different methods of load carriage. Ergonomics, 28, 197-212

Lloyd, R., Parr, B., Davies, S., \& Cooke, C. (2011). A kinetic comparison of back-loading and head-loading in Xhosa women. Ergonomics, 54(4), 380-391

McGill, S. M., Karpowicz, A., Fenwick, C. M. J., \& Brown, S. H. M. (2009a). Exercises for the torso per- 
formed in a standing posture: spine and hip motion and motor patterns and spine load. Journal of Strength and Conditioning Research, 23(2), 455-464

McGill, S. M., Marshall, L., \& Andersen, J. (2013). Low back loads while walking and carrying: comparing the load carried in one hand or in both hands. Ergonomics, 56(2), 293-302

McGill, S. M., McDermott, A., \& Fenwick, C. M. J. (2009b). Comparison of different strongman events: trunk muscle activation and lumbar spine motion, load, and stiffness. Journal of Strength and Conditioning Research, 23(4), 1148-1161

McGill, S. M., \& Norman, R. W. (1985). Dynamically and statically determined low back moments during lifting. Journal of Biomechanics, 18(12), 877-885

Moore, K. L., Dalley, A. F., \& Agur, A. M. R. (2014). Clinically Oriented Anatomy, Seventh Edition. Philadelphia: Lippincott, Williams \& Wilkins.

Piggyback New West. (2013). Piggyback New West. Retrieved from https://www.facebook.com/PiggybackNewWest/info

Robertson, D. G. E., Caldwell, G. E., Hamill, J., Kamen, G., \& Whittlesey, S. N. (2004). Research Methods in Biomechanics. Champaign, IL: Human Kinetics.
Rodriguez-Soto, A. E., Jaworski, R., Jensen, A., Niederberger, B., Hargens, A., Frank, L. R., Ward, S. R. (2013). Effect of load carriage on lumbar spine kinematics. Spine, 38(13), E783-E791

Rugby, N. (2011). All for One and One for All. Retrieved from http://www.nottinghamrugby.co.uk/news-events/ got-your-back.aspx

Schneider, H. (2009, February 17). Working out as a team: she ain't heavy, she's my other, The Washington Post, p. F3.

Sportsmail Reporter. (2011). Ramsey, Bellamy, and Bale return to Wales training ahead of England clash. Mail Online. Retrieved from http://www.dailymail.co.uk/sport/ football/article-1368789/Gareth-Bale-Craig-BellamyAaron-Ramsey-return-Wales-training.html

Vacheron, J. J., Poumarat, G., Chandezon, R., \& Vanneuville, G. (1999). Changes of contour of the spine caused by load carrying. Surgical Radiologic Anatomy, 21(2), 109-113

Wannop, J., Worobets, J., \& Stefanyshyn, D. (2012). Normalization of ground reaction forces, joint moments, and free moments in human locomotion Journal of Applied Biomechanics, 28(6), 665-676 